Proceedings of the 48th International School and Conference on the Physics of Semiconductors "Jaszowiec 2019"

\title{
Modulated Ammonia Flow \\ Low Temperature AlN Buffer LP-MOVPE Growth for High Quality AlGaN Layers
}

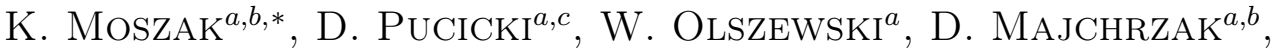 \\ J. SERAFIŃCZUK ${ }^{a, c}$ AND D. HOMMEL ${ }^{a, d}$ \\ ${ }^{a}$ Łukasiewicz Research Network - PORT Polish Center for Technology Development, \\ Stabłowicka 147, 54-066 Wrocław, Poland \\ ${ }^{b} \mathrm{~W}$. Trzebiatowski Institute of Low Temperature and Structure Research, Polish Academy of Sciences, \\ Okólna 2, 50-422 Wrocław, Poland \\ ${ }^{c}$ Faculty of Fundamental Problems of Technology, Wrocław University of Science and Technology, \\ Wybrzeże Wyspiańskiego 27, 50-370 Wrocław, Poland \\ ${ }^{d}$ Faculty of Physics, University of Wrocław, Pl. M. Borna 9, 50-204 Wrocław, Poland
}

\begin{abstract}
The structural properties of AlGaN layers deposited on high- and low-temperature grown AlN buffer were analysed. Modulated ammonia flow method of preparing AlN buffer was compared with a constant ammonia flow process using metalorganic vapour phase epitaxy. Low-temperature growth of the AlN buffer led to an increase of surface roughness, nevertheless crystal quality was comparable to the AlN buffer grown in high-temperature. Low-temperature nucleation AlN on sapphire substrates was required due to differences in thermal expansion and possible reduction of the number of dislocation. Modulated ammonia flow method provides an alternative approach for the AlN buffer growth at reduced reactor temperature.
\end{abstract}

DOI: 10.12693/APhysPolA.136.589

PACS/topics: epitaxy, low-temperature AlGaN/AlN, MOVPE, UV LED

\section{Introduction}

Aluminium gallium nitride $(\mathrm{AlGaN})$ is often used as a material in optoelectronics, especially in the deep ultraviolet (UV) region, due to its wide direct band-gap $(3.4-6.2 \mathrm{eV})$. It is mainly applied in light-emitting diodes (LEDs) and laser diodes (LDs) with emission wavelengths in the range of $230-350 \mathrm{~nm}[1-3]$. AlGaN can be doped as a $p$ - and $n$-type semiconductor in a wide band-gap spectral region and is thermally stable [4]. The typical ultraviolet LED structure consists of a substrate, AlN buffer, $n$-AlGaN layer, active region, an electron blocking layer, and $p$-AlGaN layer. To prepare such structure, a buffer with high aluminium content is required. Heteroepitaxial layers suffer from a high threading dislocations density (TDD), that originate from lattice and thermal coefficient mismatches between sapphire and AlN. Dislocations have a major impact on LED parameters, e.g. external quantum efficiency (EQE). Various methods are used to reduce TDD in the buffer layer. Epitaxial lateral overgrowth (ELO) [5], SiNx interlayers [6], or short period superlattice $(\mathrm{GaN} / \mathrm{AlN})$ [7] are applied to reduce dislocation density. Also, it is important to obtain atomically flat surface without cracking and with $\mathrm{Al}$ polarity. Many groups introduced different

*corresponding author; e-mail: karolina.moszak@port.org.pl ideas for obtaining high-quality AlN buffer layer. Hirayama et al. [4] proposed ammonia pulse-flow [8,9] multilayer AlN growth method on sapphire by metalorganic vapour phase epitaxy (MOVPE). Miyake [10] proposed magnetron sputtering of the AlN buffer and recrystaline them at high temperature $\left(1600-1700^{\circ} \mathrm{C}\right)$. This method was used as an alternative to the ELO idea for reducing costs and the number of needed technological steps. This technique was used as a novel concept for preparing UV LEDs [11].

One of the important parameters is high reactor temperature $>1100^{\circ} \mathrm{C}$ for obtaining $2 \mathrm{D}$ growth of the AlN layer. In the following experiment, we proposed a modulated ammonia flow for AlN buffer layer growth. For the study of the quality of grown samples highresolution X-ray diffraction (HRXRD), scanning electron microscopy (SEM), and atomic force microscopy (AFM) were used.

\section{Experiment}

Single-side polished $c$-plane sapphire with a miscut of $0.2^{\circ}$ toward the $a$-direction was used as a substrate for structures deposition by MOVPE. The Aixtron system contains close coupled showerhead (CCS) reactor with a $3 \times 2$ inch configuration. To investigate the influence of buffer layer on quality of $\mathrm{AlGaN}$ layers two different AlN buffers were prepared. $0.9 \mu \mathrm{m}$ thick AlN buffers were grown on sapphire: one with hightemperature AlN (HT AlN) $1250^{\circ} \mathrm{C}$ and other with 
low-temperature AlN (LT AlN) $1100^{\circ} \mathrm{C}$. The first sample (HT_AlN) was grown at high reactor temperature $T_{r}=14 \overline{90}{ }^{\circ} \mathrm{C}$, that corresponds with susceptor temperature $T_{s}=1230^{\circ} \mathrm{C}$. AlN buffer was grown with a constant ammonia flow of $6.70 \mathrm{mmol} / \mathrm{min}$ and the $\mathrm{V} / \mathrm{III} \mathrm{ra}-$ tio of 221. The second sample (LT_AlN) was grown at a reactor temperature of $1300^{\circ} \mathrm{C}\left(T_{s}=1100^{\circ} \mathrm{C}\right)$ with linearly decreasing the ammonia flow from $4.06 \mathrm{mmol} / \mathrm{min}$ to $0.36 \mathrm{mmol} / \mathrm{min}$, trimethylaluminum (TMAl) was dispensed at $0.04 \mathrm{mmol} / \mathrm{min}$ flow rate. Both samples had a similar structure and composition. The aluminium content in the following layers differs only by $2-3 \%$. According to XRD at the top of HT_AlN buffer there were $380 \mathrm{~nm}$ of $\mathrm{Al}_{0.90} \mathrm{Ga}_{0.10} \mathrm{~N}$ and $35 \overline{\mathrm{n}} \mathrm{nm}$ of $\mathrm{Al}_{0.83} \mathrm{Ga}_{0.17} \mathrm{~N}$ layers deposited in sequence (Fig. 1a). On the LT AlN buffer, there were also $380 \mathrm{~nm}$ of $\mathrm{Al}_{0.89} \mathrm{Ga}_{0.11} \mathrm{~N}$ and $350 \mathrm{~nm}$ of $\mathrm{Al}_{0.80} \mathrm{Ga}_{0.20} \mathrm{~N}$ deposited in sequence (Fig. 1b). The reflectance signal at $405 \mathrm{~nm}$ was recorded in situ using LayTec EpiTT system during the process.

(a)

\begin{tabular}{|c|}
\hline $\mathrm{Al}_{0.83} \mathrm{Ga}_{0.17} \mathrm{~N}(350 \mathrm{~nm})$ \\
\hline $\mathrm{Al}_{0.90} \mathrm{Ga}_{0.10} \mathrm{~N}(380 \mathrm{~nm})$ \\
\hline HT_AIN $(900 \mathrm{~nm})$ \\
\hline Sapphire $(430 \mu \mathrm{m})$ \\
\hline
\end{tabular}

Fig. 1. Scheme of MOVPE grown structures: (a) Al-

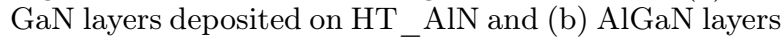
deposited on LT_AlN.

\section{Results}

To investigate the crystal structure, both samples were analysed using high-resolution X-ray diffraction (HRXRD) (Fig. 2). Symmetrical (002) and asymmetrical (015) reflections were measured. For both cases, we assume that the AlN buffer is fully relaxed to the sapphire substrate. However, AlGaN layers in both samples have various degree of relaxation to AlN buffer. AlGaN layers deposited on HT_AlN buffer are less relaxed than AlGaN layers deposited on LT_AlN buffer. A $380 \mathrm{~nm}$ thick $\mathrm{Al}_{0.90} \mathrm{Ga}_{0.10} \mathrm{~N}$ layer on an $\mathrm{HT}$ AlN buffer has $5 \%$ relaxation. The top $350 \mathrm{~nm}$ thick layer of $\mathrm{Al}_{0.83} \mathrm{Ga}_{0.17} \mathrm{~N}$ is relaxed by $9.3 \%$ to the AlN. In Fig. 3a a slight deviation between peaks positions is visible.

The second structure contains a $380 \mathrm{~nm}$ thick layer of $\mathrm{Al}_{0.89} \mathrm{Ga}_{0.11} \mathrm{~N}$ relaxed by $29.1 \%$ to $\mathrm{LT}$ AlN buffer. The $350 \mathrm{~nm}$ thick $\mathrm{Al}_{0.80} \mathrm{Ga}_{0.20} \mathrm{~N}$ layer is relaxed by $32.9 \%$. In Fig. 3b a significant shift in peak positions can be seen. Reciprocal space maps of 015 reflexes show differences between HT AIN and LT AlN buffers. Figure $3 \mathrm{~b}$ shows wider signal from $\mathrm{LT}_{-}$AlN buffer than from HT AlN (Fig. 3a). Broadening of peaks for AlGaN layers is also visible for the sample with LT_AlN buffer, which means increased crystal deformation. A higher degree of relaxation is due to the lower quality of the crystal structure and a higher number of defects. The XRD (002) rocking curve (Fig. 2) full width and half maximum (FWHM) have similar values, for LT_AlN 104.4 arcsec and $\mathrm{HT}_{\text {_ AlN }} 115.7$ arcsec.

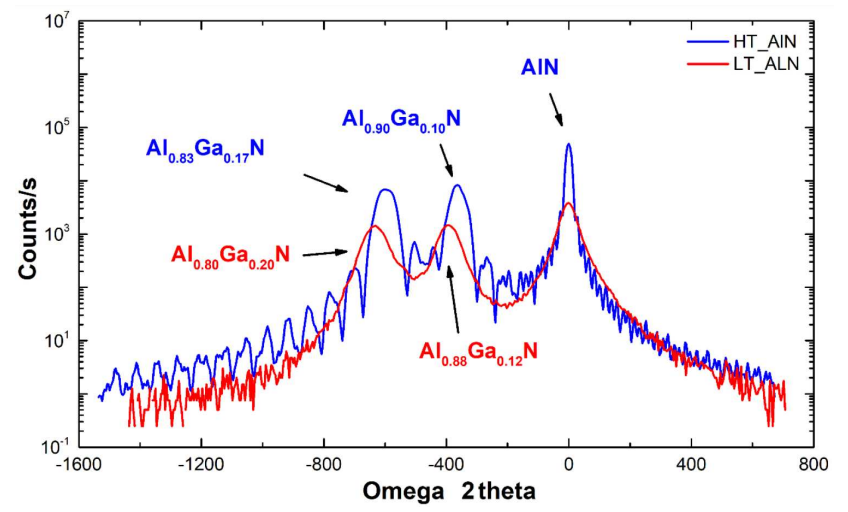

Fig. 2. XRD scans for samples with HT_AlN and LT_AlN.

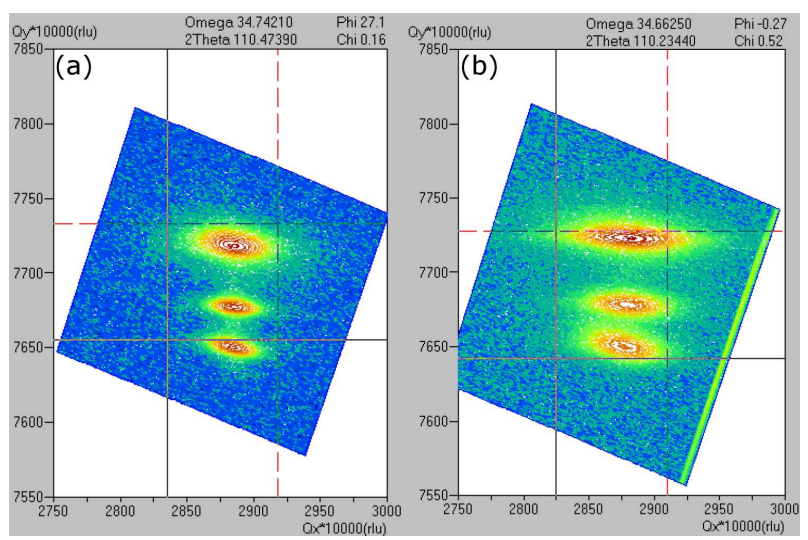

Fig. 3. Asymmetric (015) reciprocal space maps for samples (a) with HT_AlN and (b) LT_AlN.
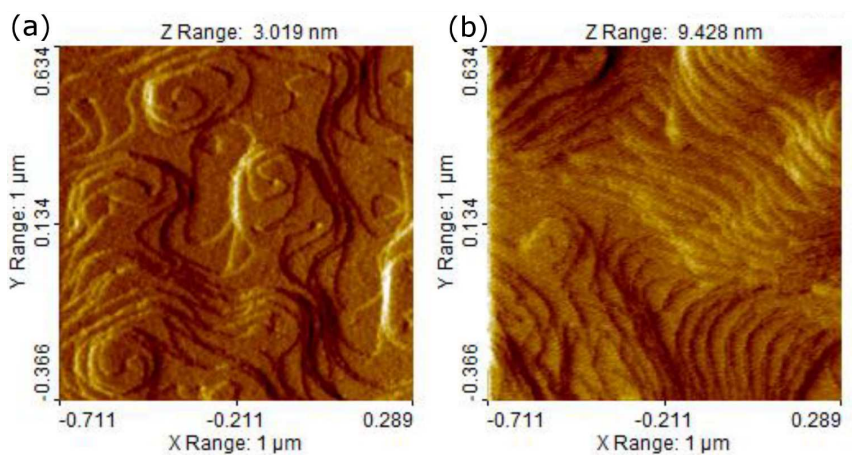

Fig. 4. AFM images of the samples with (a) HT AlN $(\mathrm{RMS}=0.64 \mathrm{~nm})$ and $(\mathrm{b})$ LT_AlN $(\mathrm{RMS}=1.68 \overline{\mathrm{nm}})$. 
The surface topography was measured with the AFM system in contact mode. The root mean square (RMS) roughness of the samples was measured on area $1 \mu \mathrm{m} \times 1 \mu \mathrm{m}$ (Fig. 4). For the structure with $\mathrm{HT}_{-} \mathrm{AlN}$ buffer, the RMS is $0.64 \mathrm{~nm}$ and is 2.6 times smaller than for the structure with LT_AlN buffer $($ RMS $=1.68 \mathrm{~nm})$. Low-temperature AlN buffer is less homogeneous. Despite small differences observed in RSM and diffraction curves, the surfaces of the two samples are comparable smooth which indicates that LT_AlN buffer satisfies the requirements of devices structures growth.
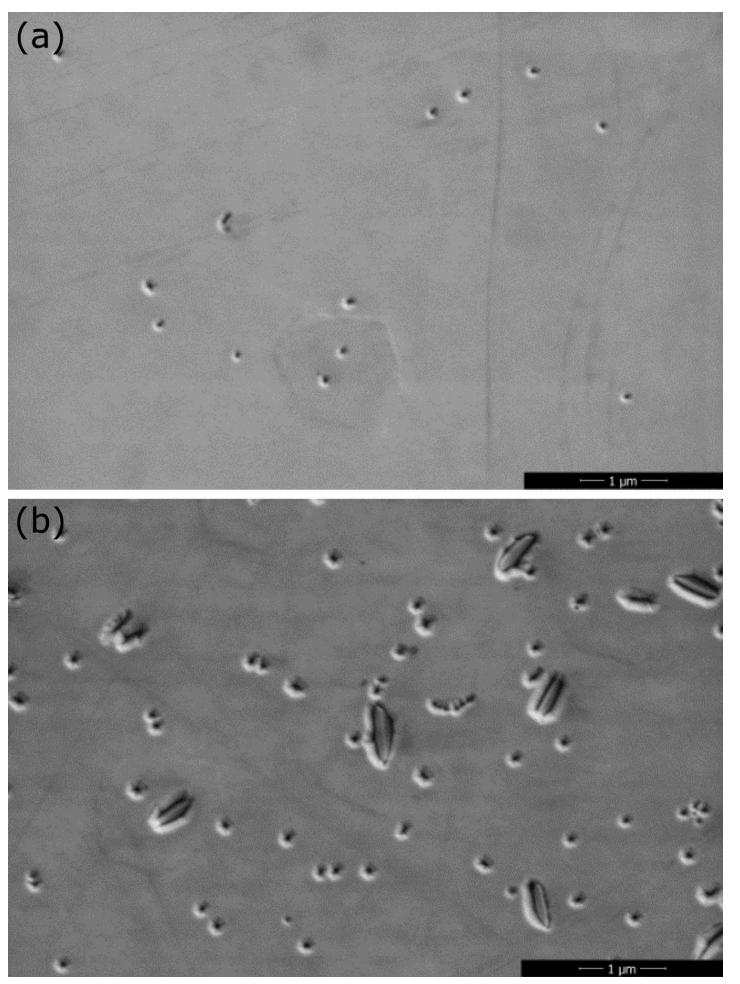

Fig. 5. SEM images of AlGaN epilayers on (a) HT_AlN and (b) LT_AlN.

Figure 5 shows surfaces of analysed structures with 25000 times magnification by scanning electron microscopy. On both surfaces, there are visible holes that match etch pits in shape. This means that at the end of the process AlGaN material is chemically etched by hydrogen. Irregular lines, created by dislocation, are also visible on both surfaces. The surface with LT AlN buffer has more defects. Dislocation density for AlN buffer layer is $4.59 \times 10^{10} \mathrm{~cm}^{-2}$, obtained from plane-view TEM, which is state of the art for AlN layers.

To investigate optical quality of both samples photoluminescence was performed at $15 \mathrm{~K}$ and with $213 \mathrm{~nm}$ excitation laser wavelength (Fig. 6). The structure with high-temperature AlN buffer has an emission peak at slightly higher energy of $5.45 \mathrm{eV}$. Despite the structure grown on HT-AlN buffer being characterized by slightly higher PL signal, the optical quality of both samples are comparable.

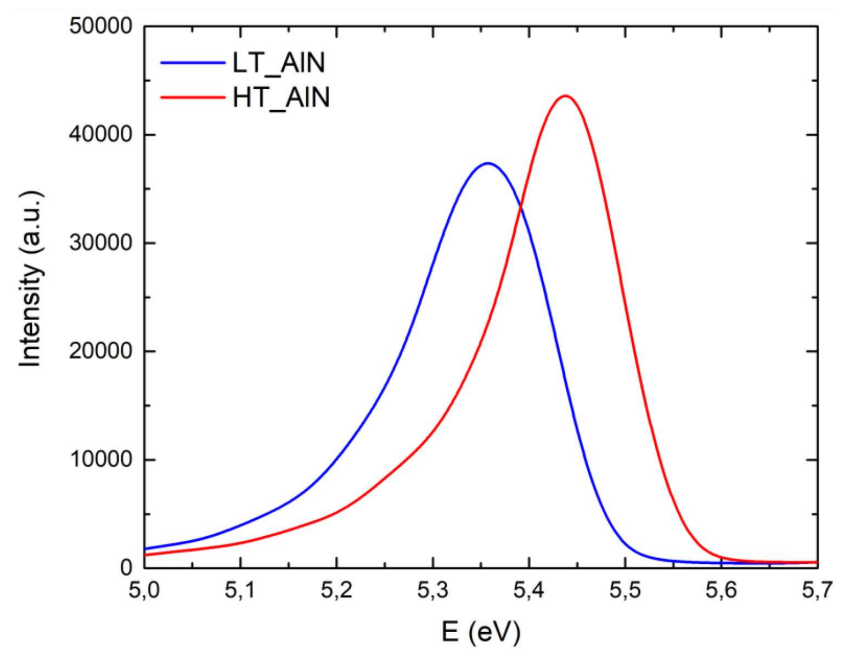

Fig. 6. Low temperature (at $15 \mathrm{~K}$ ) photoluminescence of two structures.

\section{Summary}

Two AlGaN/AlN structures grown on sapphire were investigated. In the first structure, AlGaN layers were deposited on the high-temperature AlN buffer layer. The second sample includes two AlGaN layers deposited on the low-temperature AlN buffer with a linear decrease of the ammonia flow. It was found that structure obtained during growth in high temperature has higher quality with lower surface roughness. The relationship between the quality of AlN buffer and AlGaN layers relaxation has been investigated.

Modulated ammonia flow method can be used to obtain high-quality AlN buffer layer. This method gives similar results to the standard high-temperature procedure of AlN growth. As it was presented, this method can produce buffers with a good crystalline structure. It is a promising method for the mass production growth of AlN buffer layers.

\section{References}

[1] T. Nishida, H. Saito, N. Kobayashi, Appl. Phys. Lett. 79, 711 (2001).

[2] H. Hirayama, Y. Enomoto, A. Kinoshita, A. Hirata, Y. Aoyagi, Appl. Phys. Lett. 80, 37 (2002).

[3] J.P. Zhang, A. Chitnis, V. Adivarahan, S. Wu, W. Mandavilli, R. Pachlpulusu, M. Shatalov, G. Simin, J.W. Yang, M. Asif Khan, Appl. Phys. Lett. 81, 4910 (2002).

[4] H. Hirayama, T. Yatabe, N. Noguchi, T. Ohashi, N. Kamata, Appl. Phys. Lett. 91, 071901 (2007).

[5] V. Kueller, A. Knauer, U. Zeimer, M. Kneissl, M. Weyers, J. Cryst. Growth 368, 83 (2013).

[6] K. Engl, M. Beer, N. Gmeinwieser, U.T. Schwarz, J. Zweck, W. Wegschneider, S. Miller, A. Miller, H.J. Lugauer, G. Bruderl, A. Lell, V. Harle, J. Cryst. Growth 289, 6 (2006). 
[7] H.M. Wang, J.P. Zhang, C.Q. Chen, Q. Fareed, J.W. Yang, M.A. Khan, Appl. Phys. Lett. 81, 604 (2002).

[8] P. Caban, M. Rudziński, M. Wojcik, J. Gaca, W. Strupiński, J. Cryst. Growth 414, 81 (2015).

[9] H.J. Kim, S. Choi, D. Yoo, J.H. Ryou, R.D. Dupuis, R.F. Dalmau, P. Lu, Z. Sitar, Appl. Phys. Lett. 93, 022103 (2008).
[10] H. Miyake, C.H. Lin, K. Tokoro, K. Hiramatsu, J. Cryst. Growth 456, 155 (2016).

[11] N. Susilo, S. Hagedorn, D. Jaeger, H. Miyake, U. Zeimer, C. Reich, B. Neuschulz, L. Sulmoni, M. Guttmann, F. Mehnke, C. Kuhn, T. Wernicke, M. Weyers, M. Kneissl, Appl. Phys. Lett. 112 041110 (2018). 